\title{
Development and Evaluation of Infrared Blood Pressure Monitoring System
}

\author{
Toshiya Arakawa ${ }^{\mathrm{a},}$, Noriaki Sakakibara ${ }^{\mathrm{b}}$, Shinji Kondo ${ }^{\mathrm{b}}$ \\ aAichi University of Technology, 50-2, Manori,Nishihasama-cho, Gamagori-city, Aichi 443-0047, Japan \\ bANDS Inc., 93-3, Nakamaeda, Igaya-cho, Kariya-city, Aichi 448-0001, Japan \\ *Corresponding Author: arakawa-toshiya@aut.ac.jp
}

\begin{abstract}
Blood pressure monitors have been developed and sold widely and are in wide use throughout society, but these are primarily what are termed non-invasive monitors. The monitors currently available have some disadvantages including discomfort for the patient caused by painful cuff inflation, which may actually influence blood pressure readings, and the unfeasibility of continuous or semi-continuous blood pressure monitoring due to the necessity for repetitive cuff inflation and deflation. Cuffless blood pressure measuring systems have been developed which help to solve these problems, and in this paper, infrared cuffless blood pressure monitoring system and its effectiveness and practicality are introduced. Their measurement principles are described and their performance evaluation is also explained. The primary disadvantage of steering-type blood pressure monitoring systems is found to be accuracy; infrared blood pressure monitoring systems currently suffer from some time lag in reporting of results.
\end{abstract}

Keywords: Hypertension, Blood pressure, Infrared blood pressure monitoring system

\section{Introduction}

There is a growing awareness of the importance of lifestyle in achieving and maintaining good health [1]. As such awareness increases, the basic lifestyles of all people are changing to incorporate better-rounded lifestyles. Not only is this important at a personal level, but it is also related to national strategies; in response, new business development related to healthcare has appeared around the world. One health area that has received great interest is that of high blood pressure; it can cause major diseases and ailments such as strokes and heart and kidney diseases [2,3]. Considering the rapid progression of the aging population and a Westernized diet $[4,5]$, it is becoming increasingly important to prevent the occurrence of hypertension in Japan as in the rest of the world. Hypertension, a major contributor to cardiovascular disease (CVD) including heart disease and stroke, is one of the leading contributors to the global burden of disease and is a growing public health problem worldwide [6]. In the U.S., about 75.2 million adults (one in every three) had hypertension during 20132014 [7]. In 2014, hypertension was listed as a primary or contributing cause of 427,631 American deaths, and heart disease and stroke were the first-and fifth-leading causes of death respectively [8].

A study based on analysis data from the 2011 to 2014 National Health and Nutrition Examination Survey ( $\mathrm{n}=$ 9623) shows that, according to the criteria from the 2017 American college of Cardiology/American Heart Association (ACC/AHA) and the Seventh Report of the Joint National Committee on Prevention, Detection, Evaluation, and Treatment of High Blood Pressure (JNC7) guidelines, the crude prevalence of hypertension among American adults was $45.6 \%$ (95\% confidence interval (CI): $43.6 \%-47.6 \%$ ) and $31.9 \%$ (95\% CI: $30.1 \%-33.7 \%)$, respectively. In addition, antihypertensive medication was recommended for $36.2 \%$ (95\% CI: $34.2 \%-38.2 \%$ ) and $34.3 \%$ (95\% CI: $32.5 \%-36.2 \%)$ of American adults, respectively [9]. Another report shows that one in three adults in the United States has high blood pressure, and half of them do not have it under control [10].

Another study of the relation between hypertension and economic loss showed that the total cost of treating hypertension in the United States in 2030 will be US\$50.3 billion-US\$47.2 billion in direct medical costs, and US\$13.1 billion in indirect costs owing to lost productivity 
related to morbidity and mortality [11]. Based on this study, the total direct cost of hypertension is followed by coronary heart disease (CHD; US\$61.2 billion) and the total indirect costs of lost productivity is followed by CHD (US $\$ 129.9$ billion) and stroke (US $\$ 51.2$ billion). Thus, it is found that hypertension significantly leads to decreased productivity and economic loss.

Besides decreasing productivity and economic loss, hypertension is also a major cause of traffic accidents [12]. One study shows that hypertension is the most common chronic pathology found in traffic offenders [13]. Another study reported that hypertension is highly prevalent among bus drivers, a group responsible for the safety of many others on the road [14]. It is therefore important for professional drivers, like bus drivers, to manage their own health condition daily, and to be aware that they should not drive without rest or when they are unwell. In addition, it is desirable to develop a system that manages the driver's health and monitors sudden spikes in blood pressure, providing them the opportunity to safely pull off the road before a health crisis occurs, thereby decreasing the number of traffic accidents and ensuring the safety of the driver and their passengers. [12].

A previous report [10] also stated that the AHA and other organizations have called for greater use of home blood pressure monitoring; however, their use is not widespread. One reason is that insurance coverage for such programs still lags, and another is that full-fledged efforts such as those established in the state of Minnesota could cost US $\$ 1350$ per person. However, this report also states that everyone can buy a good home blood pressure monitor currently available from a pharmacy or online merchant from between US\$50 to US\$100; this would make checking blood pressure twice a day easy to accomplish for everyone.

These inexpensive non-invasive blood pressure monitors based on cuff occlusion are in wide use both inside and outside of care facilities [15]. However, they have some disadvantages including discomfort for the patient because of painful cuff inflation (which may also influence blood pressure outcome) and the unfeasibility of continuous or semi-continuous blood pressure monitoring due to the necessity for repeated cuff inflation and deflation [12]. They are not very practical for use in constant monitoring during driving or in monitoring of healthy people in their daily routines.

As a response to finding a more practical method of providing regular blood pressure monitoring, non-invasive cuffless blood pressure measurement devices have begun to be developed and mass-produced. In fact, the IEEE published a standard for wearable cuffless blood pressure measuring devices systems that can measure blood pressure based on pulse wave propagation time (IEEE1708 certified on 26 August 2014) [16]. Our survey shows that the development of cuffless blood pressure measurement monitors has been thriving since the standard was published and the worldwide proliferation of cuffless blood pressure measurement monitors has increased from the point of Google patents [12].

In this manuscript, an infrared cuffless blood pressure monitoring system is developed, and their efficiency and usability are discussed. In our previous study, a steering-type blood pressure monitoring system $[16,17]$ was developed. However, steering-like blood pressure monitoring systems are specialized for use in-vehicles, thus it is necessary to consider versatility in order to provide new technology for a wider portion of the population. In addition, if only in-vehicle use is considered, users cannot choose and buy steering-wheel based blood pressure system as optional parts after they buy a vehicle, making it quite inconvenient. Thus, an infrared blood pressure monitoring system that can be attached as a small shield over vehicle gauges was developed. If these infrared blood pressure monitoring systems are mass-produced, users can buy them and easily attach them to their vehicle.

\section{Materials and methods}

The infrared blood pressure monitoring system developed has a transmitter and receiver. The transmitter emits infrared light onto a person and the receiver receives the weak reflected infrared light from a person's skin. The reflected infrared light hits the receiver, its signal-is processed through an amplifier and a filter circuit, then the reflected infrared light is output as detected signal (called a continuous pulse wave). The signal changes based on the relative change of blood flow, which of course is based on the volume change of blood.

The flowchart shown in Figure 1 illustrates how calculation of blood pressure is performed using this information. First, calibration of the blood pressure value must be done. The user's systolic blood pressure value $P_{s}$ and diastolic blood pressure value $P_{d}$ are set manually, 
and average blood pressure value $P_{m}$ is calculated. Then, stability of the continuous pulse wave is detected, and this is calculated as standard pulse wave area $V_{0}$. Next, the coefficient of average blood pressure, the ratio of systolic blood pressure and diastolic blood pressure as well as the ratio of diastolic blood pressure are calculated as calibration value based on from equation (1) to (3):

$$
\begin{gathered}
L=\frac{V_{0}}{P_{m}} \\
O=\frac{P_{S}}{P_{m}} \\
P=\frac{P_{d}}{P_{m}}
\end{gathered}
$$

where $L$ is coefficient of average blood pressure, $O$ is the ratio of systolic blood pressure value and average blood pressure value and $P$ is the ratio of diastolic blood pressure value and average blood pressure value.

After calibration, values $L, O$ and $P$, are calculated and the actual blood pressure value is measured.

At actual measurement, $V_{0 n}$, which is the pulse wave area of every heartbeat based on the continuous pulse wave, is calculated using the same method as $V_{0}$, which is the standard pulse wave area. Next, the average blood pressure value $P_{m n}$ (this is called average blood pressure of every heartbeat) from equation (4) based on pulse wave area and coefficient of average blood pressure every heartbeat.

$$
P_{m n}=\frac{V_{0 n}}{L}
$$

After calculation of average blood pressure of every heartbeat, maximum blood pressure $P_{s n}$ and minimum blood pressure $P_{d n}$ is calculated from equation(5) and (6) based on the ratio of maximum blood pressure and average blood pressure, the ratio of minimum blood pressure and average blood pressure, and average blood pressure.

$$
P_{s n}=\frac{P_{m n}}{O}
$$

$$
P_{d n}=\frac{P_{m n}}{P}
$$

The developed infrared blood pressure monitoring system is shown in Figure 2. Figure 3 shows block diagram of the developed infrared blood pressure monitoring system.

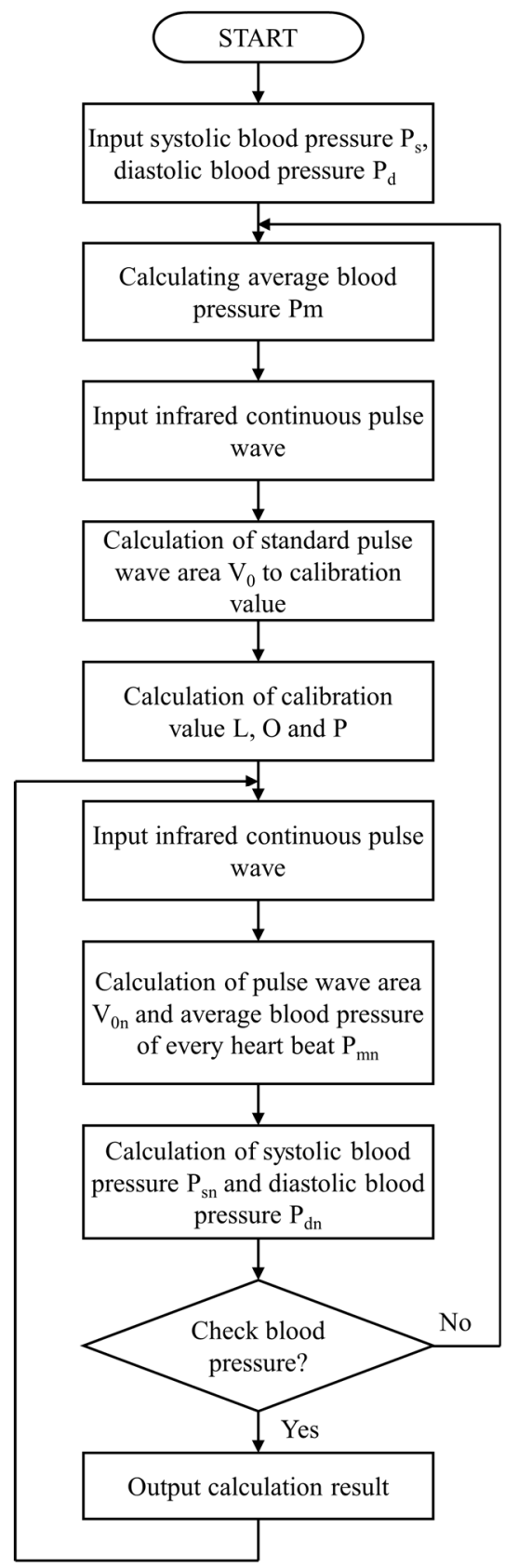

Figure 1. Flowchart of calculating blood pressure

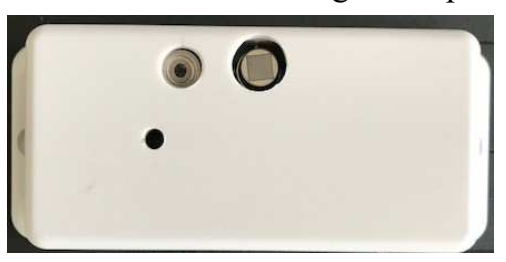

-

Figure 2. The developed blood pressure monitoring system 


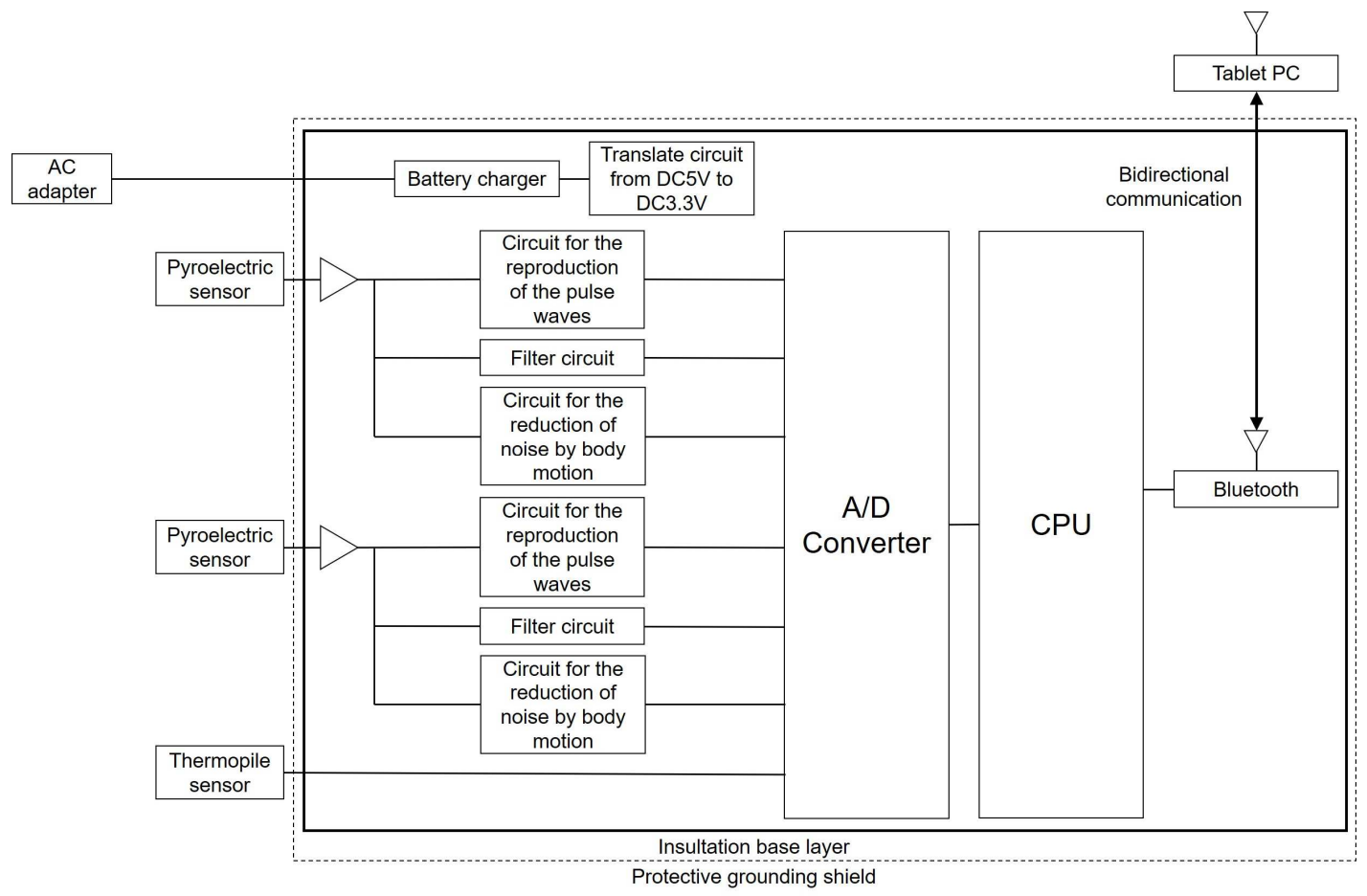

Figure 3. Block diagram of the developed blood pressure monitoring system

\section{Experiment and consideration}

Here, the experiment was to evaluate the effect of time lag when using infrared blood pressure monitoring system in vehicles. A driving simulator, DS-nano- (Advanced Solutions Technology Japan), was used for this experiment. This driving simulator has a real-vehicle-like exterior and interior. The developed blood pressure monitoring system was attached to a small shield over the driving simulator's gauges. Nine male participants (from 21 to 50 years old) joined this experiment. All participants were briefed on the experiment and informed consent was obtained from all participants.

All experiments required the participants to drive in an urban-area-like simulation environment, which has many intersections of low visibility. They were asked to drive at approximately $50 \mathrm{~km} / \mathrm{h}$ for about $4 \mathrm{~min}$. After driving of approximately $3 \mathrm{~min}$, a vehicle appeared to run into the road from a hidden location (Figure 4). Here, the direction from which the vehicle ran (whether from left or from right) was random. The participant's blood pressure and driver behavior (velocity, steering angle and brake pressure) were measured. For the purpose of evaluating the time lag, a contact type continuous blood pressure monitoring system ( $\mu$ BP-mp, KANDS, Inc.) was also attached to participant and his blood pressure was measured. In addition, perspiration sensors were also attached to their left and right thumbs to detect perspiration that occurred by the sudden appearance of the additional vehicle. Figure 5 shows the details of the experiment. Figure 5(a) shows a participant's driving situation and Figure 5(b) shows an outline of the driving simulator as it records the participant.

The changepoint method was applied in order to detect the first change point from the unexpected vehicle's first appearance in blood pressure time-series data. The changepoint method considers $k$ as the number of change point and $\tau$ as vector which consists of some change point. Now, consider $\tau=\left(\tau_{0}, \tau_{1}, \cdots, \tau_{k+1}\right), l(\cdot)$ as $\log$ likelihood and $\lambda$ as penalty. Here, likelihood ratio is considered as following:

$$
\min _{k, \tau}\left(\sum_{i=1}^{k+1}\left[-l\left(z_{\tau_{i}-1: \tau_{i}}\right)\right]+\lambda k\right)
$$

where $z_{t}$ means time-series data of which average value is change in time, so,

$$
z_{t} \mid \theta_{t} \sim N\left(\theta_{t}, 1\right)
$$

Change point can be detected according to value of $\lambda$. Many change points are detected when value of $\lambda$ is small, and vice versa. In this paper, changepoint package of 
R 3.6.0 was used to detect change points [18]. Figure 6 shows that an example of detecting change points of a participant's average blood pressure time-series data. In Figure 6, red vertical lines mean detected change points.

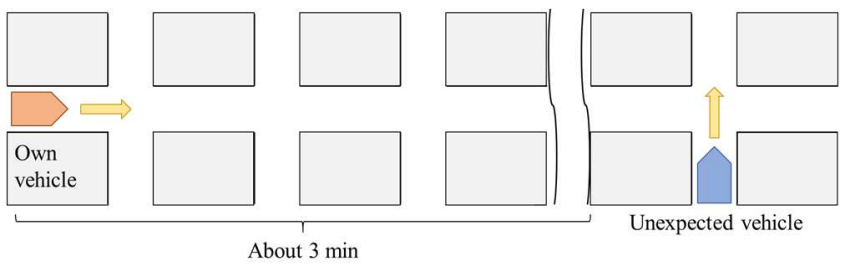

Figure 4. Driving situation. After about 3-min driving, an unexpected vehicle appears.
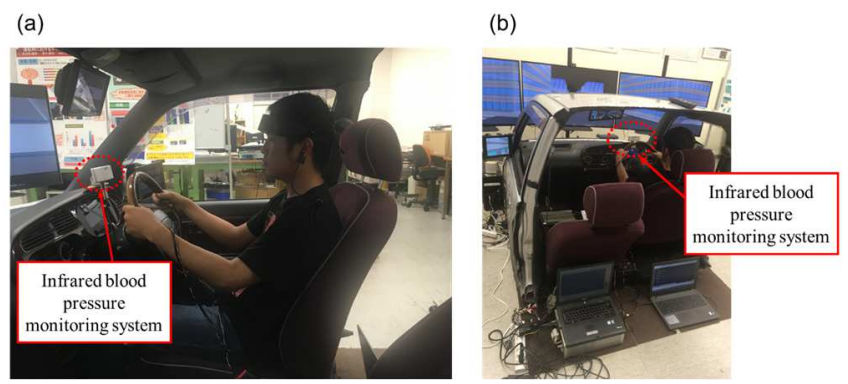

Figure 5. Situation of experiment. (a): participant's driving situation and (b): outline of driving simulator on participant's driving.

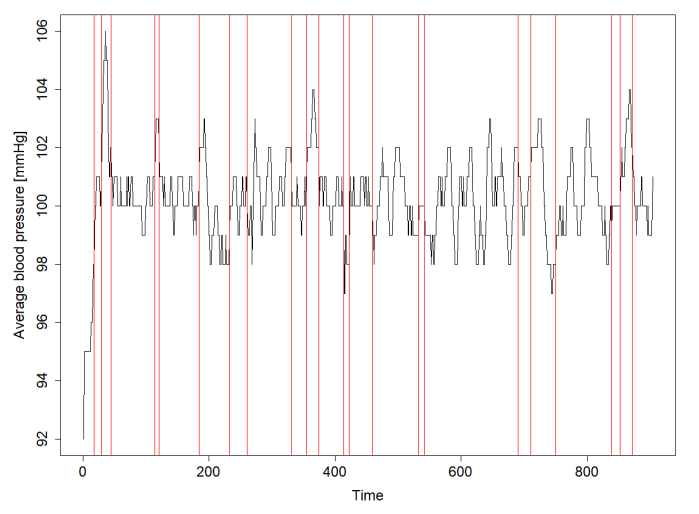

Figure 6. An example of detecting change points by the changepoint method $(\lambda=11)$. Red vertical lines mean detected change points.

First, it was verified whether participants were really surprised at the appearance of the unexpected vehicle and whether their blood pressure increased. It is known that perspiration shows a person's tension, thus the timing of perspiration from the unexpected vehicle shows whether participants were really surprised at the appearance of another vehicle. Figure 6 shows the average time $\pm 1 \mathrm{SD}$ of all participants to increase the perspiration value from the point of the appearance of the vehicle based on the changepoint method. In Figure 6, left means time to increase the perspiration value of the left thumb, right means the time to increase the perspiration value of the right thumb, and $\min (\mathrm{L}, \mathrm{R})$ means the minimum time of "left" and "right." From Figure 7, all participants expressed a perspiration response, thus it is shown that all participants were surprised at the sudden appearance of another vehicle.

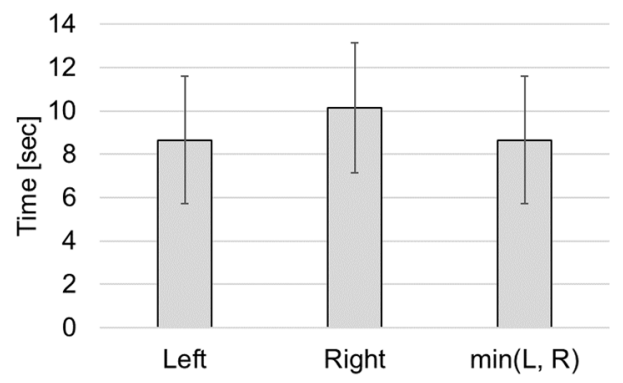

Figure 7. Average time $\pm 1 \mathrm{SD}$ of all participants to increase the perspiration value from the point of appearing unexpected vehicle

The timing that the average blood pressure increased from the point of an unexpected vehicle was first seen as it appeared was calculated. The changepoint method was applied in order to detect the timing. From the result of the calculation by the changepoint method, the average increase in time $\pm 1 \mathrm{SD}$ of all participants is shown in Figure 8. Here, in Figure 8, “ $\mu$ BP-mp" means the timing based on blood pressure measured with contact type continuous blood pressure monitoring system ( $\mu$ BP-mp) and "IR" means the timing based on blood pressure measured with infrared blood pressure monitoring system.

Time lag to detect blood pressure is important because this infrared blood pressure measurement system is a non-contact measurement system. However, it was found that the detection time by the developed systems is about $2.7 \mathrm{sec}$ longer than that for contact continuous blood pressure measurement systems, which is not a significant difference. Thus, delay of the detection timing of blood pressure increase may not affect the actual operation if psychological tension of a vehicle's sudden appearances is detected with infrared blood pressure measuring systems.

\section{Conclusion}

In this paper, two blood pressure monitoring systems were introduced: the first is a steering-type blood pressure monitoring system and the second is infrared blood pressure monitoring system. Both have advantages particularly for the drivers of vehicles; however, there are 


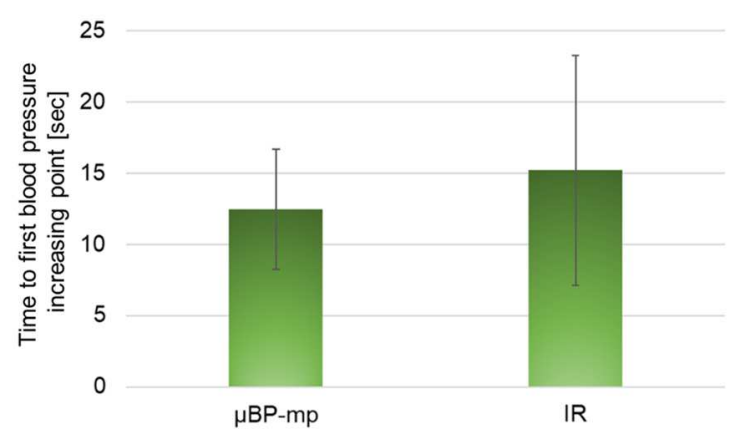

Figure 8 . Average increasing time $\pm 1 \mathrm{SD}$ of all participants

still many problems that need to be addressed to achieve downsizing and improvement of accuracy. However, these blood pressure measurements could dramatically support improved lifestyles and dynamically increase driver safety.

\section{Acknowledgment}

The authors would like to thank Misaki Design LCC. for developing the cabin of the driving simulator.

\section{References}

(1) Meguro S (2001) Marketing in health care-Strategic insight into new business development. Reitaku Int. J. Econ. Stud. 19:56-71. (In Japanese)

(2) Moser M (1992) High blood pressure. In Yale University School of Medicine Heart Book. William Morrow \& Co.: New York, NY, USA

(3) Stroke Association. High Blood Pressure and Stroke. Available online: https://www.stroke.org.uk/sites/default/files/high_blood pressure_and_stroke.pdf. Accessed 16 December 2017.

(4) Monge A, Lajous M, Ortiz-Panozo E,et al (2018) Western and Modern Mexican dietary patterns are directly associated with incident hypertension in Mexican women: A prospective follow-up study. Nutr. J.: 17, 21.

(5) Rai SK, Fung TT, Lu N, et al (2017) The Dietary Approaches to Stop Hypertension (DASH) diet, Western diet, and risk of gout in men: Prospective cohort study. BMJ 357: j1794. [CrossRef] [PubMed]

(6) Constant AF, Geladari EV, Geladari CV. (2016) The economic burden of hypertension. Chapter 21. In: Andreadis EA, editor. Hypertension and Cardiovascular Disease. Switzerland: Springer International Publishing; https://doi.org/10.1007/978-3-319-39599-9_21.

(7) Merai R, Siegel C, Rakotz M et al (2016) CDC grand rounds: a public health approach to detect and control hypertension. MMWR Morb Mortal Wkly Rep. ;65(45):1261-1264. https://doi.org/10.15585/mmwr.mm6545a3.

(8) CDC, National Center for Health Statistics (2016) Multiple Cause of Death 1999-2015. CDC WONDER online database. http://wonder.cdc.gov/mcd-icd10.html. Accessed June 10, 2017

(9) Muntner, P; Carey RM; Gidding Set al (2018) Potential US population impact of the 2017 American College of Cardiology/American Heart Association high blood pressure guideline. J. Am. Coll. Cardiol., 24414.

(10) Godman H (2018) Checking Blood Pressure at Home Pays off. Available online: https://www.health.harvard.edu/blog/checking-blood-pr essure-at-home-pays-off-201307036436 Accessed 1 June 2018

(11) William JE (2003) The economic impact of hypertension. J. Clin. Hypertens. 5: 3-13.

(12) Arakawa T (2018) Recent Research and Developing Trends of Wearable Sensors for Detecting Blood Pressure, Sensors, 18(9), 2772 DOI: 10.3390/s18092772.

(13) Saei A, Rahmani A, Ebadi Aet al (2018) Traffic Accidents and Health of the Driver, Trauma Mon., 23(2):e12963, 1-12 DOI: 10.5812/traumamon.12963.

(14) Sadri G (2015) A Model of Bus Drivers' Diseases: Risk Factors and Bus Accidents. Iran J Med Sci.;27(1):3941

(15) Schoot TS, Weenk M, van de Belt TH et al (2016) A new cuffless device for measuring blood pressure: A real-life validation study. J. Med. Int. Res. 18, e85.

(16) Arakawa T, Sakakibara N, Kondo S (2018) Development of non-invasive steering-type blood pressure sensor for driver state detection. Int. J. Innov. Comput. Inf. Control 14: 1301-1310.

(17) Arakawa T, Kaminaga K, Sakakibara N et al (2016) Development and evaluation steering-type blood pressure measuring monitor based on plethysmography. In Proceedings of the IIAE Annual Conference 2016, Kyoto, Japan, 8-12 September 2016; pp. 3-4. (In Japanese)

(18) Killck R, Haynes K, Eckleyet al (2019) package 'changepoint', https:/cran.r-project.org/web/packages/changepoint/ch angepoint.pdf. Accessed 28 September 282019 